\section{Etiología del infarto renal. Revisión sistemática de 1.582 casos de la literatura}

\author{
ANA CARINA PIZZAROSSA ${ }^{1}$, VALENTINA MÉROLA ${ }^{1}$
}

\section{Etiology of renal infarction. A systematic review}

\begin{abstract}
Background: Renal infarction is a rare and usually underdiagnosed entity. Aim: To study the etiology of renal infarction in published series. Material and Methods: A systematic review was carried out selecting 28 series that included 1582 patients. Results: The proposed cause was cardiac or aortic embolism in 718 cases (45\%), an arterial injury in 253 (16\%), prothrombotic factors in 146 (9\%) and other causes in 79 (5\%). 291 cases were classified as idiopathic (18.4\%). Atrial fibrillation was present in 542 of the 718 patients with cardiac or aortic embolism. Conclusions: The main cause of renal infarction is cardiac or aortic embolism and among this group, most cases are due to atrial fibrillation. One out of five cases is labeled as idiopathic.
\end{abstract}

(Rev Med Chile 2019; 147: 891-900)

Key words: Atrial Fibrillation; Embolism; Infarction; Kidney. 'Clínica Médica C, Facultad de
Medicina, Universidad de la
República. Montevideo, Uruguay.
Trabajo no recibió
financiamiento.
Los autores declaran no tener
conflictos de interés.
Recibido el 21 de octubre de
2018, aceptado el 24 de mayo
de 2019.
Correspondencia a:
Dra. Ana Carina Pizzarossa
Soca 1389 apto 104, esquina
Silvestre Blanco. CP 11300
Montevideo, Uruguay.
carina491986@gmail.com
E l infarto renal resulta de la disrupción aguda del flujo en las arterias renales o sus ramas. La información epidemiológica que tenemos acerca de esta entidad se basa en reportes y series de casos.

Es una entidad rara pero a la vez subdiagnosticada. Su escasa frecuencia se evidencia en trabajos como el de Hoxie et al que en 1940 reporta que de 14.411 autopsias realizadas, la incidencia del infarto renal era de $1,4 \%$. Mientras que en la serie de Paris et al de 2006, se observó que de los 250.000 pacientes que concurrieron a Emergencia en 4 años solo 17 presentaron infarto renal $(0,007 \%)^{1,2}$.

El subdiagnóstico también ha sido evidenciado por la literatura. En el ya nombrado trabajo de Hoxie se destaca que de los 205 casos encontrados en las autopsias, sólo 2 habían sido diagnosticados en vida. Una de las causas de este subdiagnóstico y/o retraso diagnóstico (de 1 a 6 días según las series) es la variedad de diagnósticos diferenciales que se plantean frente a un dolor unilateral en flanco. Entre ellos se destacan: cólico nefrítico y pielonefritis. Otros diagnósticos diferenciales son: cuadro agudo de abdomen y menos frecuentemente endocarditis y síndrome coronario agudo. Los elementos de sospecha diagnóstica para infarto renal son: dolor en flanco unilateral en paciente con riesgo aumentado de evento tromboembólico (por su relación con patologías cardioaortoembólicas), LDH aumentada, PCR aumentada, leucocitosis y micro y macrohematuria. En comparación con el cólico nefrítico y la pielonefritis aguda, solo en el caso del infarto renal se observaría una $\mathrm{LDH}$ aumentada. Se ha reportado que hasta $90,5 \%$ de los pacientes con infarto renal tienen LDH aumentada ${ }^{3}$.

Sin embargo, el punto de inflexión en el diagnóstico del infarto renal fue el comienzo del uso de la tomografía computada con contraste a fines de 1970 y popularizada en las décadas de 1980 y $1990^{4}$. Fue así que el número de pacientes reportados aumentó notoriamente. Por lo que, el primer estudio a solicitar frente a un paciente con dolor en un flanco sería una tomografía de aparato urinario sin contraste ya que es el método de elección para valorar a la litiasis renal que como se dijo es uno de los principales diagnósticos diferenciales del infarto renal y es mucho más frecuente que este. Si no se observa alteración por este método, el estudio que confirma el diagnóstico de infarto 
renal es la tomografía computada con contraste de aparato urinario. Este estudio ha desplazado a la angiografía renal por ser más fácil y accesible de realizar. En algunos casos se ha utilizado para diagnosticar infarto renal a la resonancia nuclear magnética y al centellograma de perfusión renal. El tratamiento de la entidad es el tratamiento de la enfermedad de base y se plantearía una terapia endovascular cuando el diagnóstico es precoz y en pacientes seleccionados. Se ha reportado que hasta $40 \%$ de pacientes ha desarrollado injuria renal aguda $^{3}$. Por lo tanto, el dolor en flanco unilateral en pacientes con riesgo tromboembólico debería levantar la sospecha de infarto renal. En ese contexto debería incorporarse en la evaluación inicial la LDH puesto que su elevación aumenta el índice de sospecha, lo que conduciría rápidamente a la realización de una tomografía y se acortarían los tiempos diagnósticos.

Si bien el subdiagnóstico continúa siendo un problema, actualmente el principal desafío quizás sea determinar el mecanismo debido a las implicancias terapéuticas (ciertas etiologías como la fibrilación auricular tienen indicado anticoagular y en otras está contraindicado).

Es en este contexto que se realizó la siguiente revisión acerca de la etiología del infarto renal en vistas a generar una propuesta para el estudio etiológico del mismo.

El objetivo primario de esta investigación es determinar la etiología del infarto renal. El objetivo secundario de la misma es generar un protocolo de diagnóstico etiológico para el infarto renal.

\section{Metodología}

\section{Estrategia de búsqueda}

Se realizó una búsqueda incluyendo las siguientes bases de datos: Medline (interfase Pubmed), SciELO de acuerdo a los siguientes criterios.

Palabras clave: renal and infarction (MESH term); infarto and renal.

Criterios de inclusión de las series: 1) redactadas en inglés o español; 2) con pacientes mayores de 14 años; 3) inclusión de 5 o más pacientes, 4) estudio de etiología de infarto renal; 5) series que no se limitaran a una sola etiología de infarto renal; 6) diagnóstico antemorten; 7) diagnóstico con tomografía computada, centellograma de perfusión renal, resonancia nuclear magnética y/o angiografía.
Criterios de exclusión: 1) diagnóstico postmorten; 2) diagnóstico con otros métodos no citados en punto 7 de criterios de inclusión.

En los artículos seleccionados se estudiaron tres variables: edad, sexo y etiología.

Con respecto a la etiología se dividieron en las categorías propuestas por Bourgault et al y Faucon et al: cardioaortoembolia, injuria arterial renal, protrombótica e idiopática ${ }^{3,5}$. Debido a la heterogeneidad de criterios para asignar la etiología en las distintas series, se adicionó la categoría de otros.

La definición de etiología cardioaortoembólica incluye: fibrilación auricular, valvulopatías, cardiomiopatía con trombo cardiaco, endocarditis y trombo mural de aorta supra-renal. La injuria arterial renal incluye: aterosclerosis, displasia fibromuscular, hematoma disecante, extensión de disección aórtica a la arteria renal, enfermedad disecante o aneurismática multifocal.

En la categoría otros quedan incluidos: trauma, post-operatorio o post-procedimiento, de causa autoinmune y miscelánea. Es de destacar que en varias series en la categoría de injuria arterial renal están incluidas las causas traumáticas y postoperatorias.

El grupo de causa protrombótica incluye estados protrombóticos hereditarios y adquiridos (entre los que se incluye cáncer). El grupo idiopático incluye a todos los pacientes en que luego de un estudio exhaustivo por parte de los investigadores originales no se pudo otorgar una causa al infarto renal.

El análisis estadístico fue realizado con EPI INFO 7.2. Las variables se expresaron en porcentaje.

\section{Resultados}

\section{Análisis de datos}

De los 76.683 artículos que arrojó esta búsqueda (76.538 en Medline y 145 en SciElo) realizada en los días 23-27 de octubre de 2017, se procedió a filtrar los artículos por el título, idioma y valorando en el resumen si incluían más de 5 casos. De esta forma se seleccionaron 42 series, cuyos resúmenes fueron estudiados por 2 revisores independientes aplicando los criterios planteados anteriormente. Se descartaron 3 artículos por tener solo diagnósticos postmorten o por diagnósticos solo basados en datos clínicos y pielografía ${ }^{1,6,7}$. Tres series por solo incluir infartos renales de 1 etiología, siete porque 
no incluían el estudio etiológico y uno porque había una publicación posterior del mismo hospital que incluía los mismos $\operatorname{casos}^{2,8-17}$ (Figura 1).

Finalmente, 28 artículos cumplieron los requisitos expuestos en el párrafo anterior. Estas series abarcan 1.582 pacientes.

De las 28 series, 26 eran retrospectivas, en 2 había seguimiento prospectivo de pacientes (Bolderman y Rhee) y la serie de Bertolotto fue prospectiva (en ella se buscaba valorar la utilidad del diagnóstico por medio de ultrasonografía mejorada con contraste $)^{18-20}$.

A continuación, se analiza en la Tabla 1 los criterios de exclusión adicionales utilizados por los diferentes investigadores. Los criterios de exclusión del resto de las series coinciden con los criterios propuestos en este trabajo.

\section{Extracción de datos}

Se extrajeron los datos relevantes de los 28 artículos seleccionados incluyendo: tamaño de la muestra, características demográficas de los pacientes, etiología del infarto renal.

\section{Características demográficas}

Con respecto a la edad media de las series fue de 60,33 $\pm 14,93$ (591 pacientes).

La mayoría de pacientes 945/1.570 (60,2\%) eran del sexo masculino. Corea del Sur es el país con más series publicadas (6), seguido por Estados Unidos (5), Taiwán (4), Japón (2), Francia (2), Turquía (2), Italia (2), Israel (2), España (1), Bélgica (1) y Austria (1). Si se ordenaran según el número de pacientes incluidos en las series, Corea del Sur encabezaría nuevamente el listado con 834 pacientes, Francia con 280 pacientes, Taiwán con 118, Japón con 72, España 62, Estados Unidos con 50 , Israel con 49, Italia 45, Turquía 28, Bélgica 27 y Austria 17.

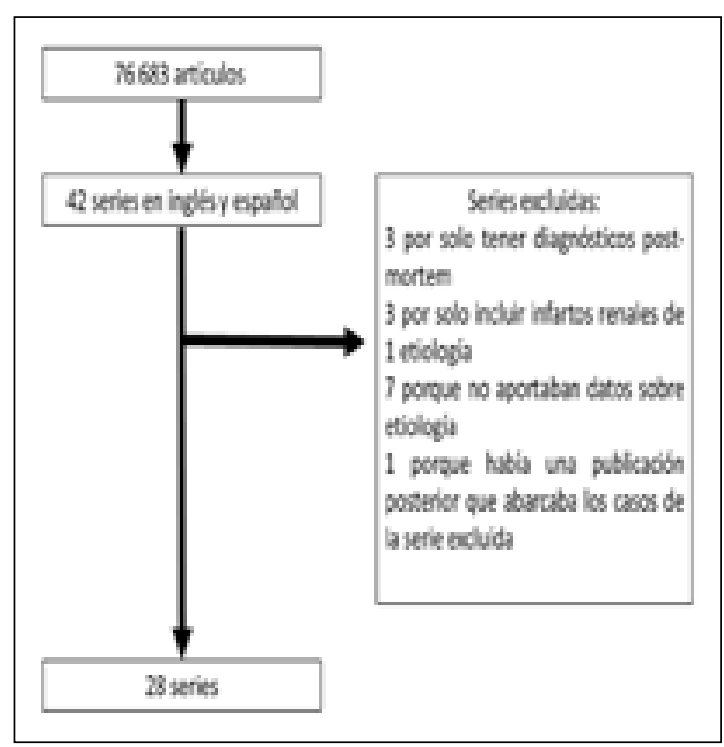

Figura 1. Selección de series.

\section{Características etiológicas}

El método por el que los diferentes autores definen la etiología del infarto renal es variado:

\section{Grupo en que se detalla cómo se evalúan pacientes}

- Faucon et al. realiza ECG, ecocardiograma transesofágico, Holter para valorar causa cardioembólica. Realiza TC de otros territorios arteriales (carótidas, polígono de Willis) para valorar lesiones en otros territorios que apoyen el diagnóstico de displasia fibromuscular (su diagnóstico se tiene que apoyar en la evidencia de lesiones en varios territorios arteriales) y de la enfermedad arterial disecante o aneurismática multifocal (su diagnóstico asocia una lesión arterial renal con un aneurisma o disección

Tabla 1. Criterios de exclusión adicionales ${ }^{4,5,18,21-27}$

\begin{tabular}{|ll|}
\hline Antopolsky & Se excluye infarto renal de causa traumática y post-operatorio \\
\hline Bolderman & Se excluye infarto renal por shock, iatrogénico y por trauma \\
Bourgalt & Se excluye infarto renal post-trasplante, post cirugía vascular y angioplastia percutánea o transluminal \\
\hline Caravaca-Fontán & Se excluye infarto renal en trasplantados \\
\hline Huang & Se excluye infarto renal de causa traumática y de menores de 14 años \\
Kwon & Se excluye infarto renal en pacientes con enfermedad renal crónica previa y menores de 20 años \\
\hline Lessman & Se excluye infarto renal de causa traumática o post cirugía o por embolia aterotromboembolica \\
Yang & Se excluyen pacientes menores de 18 años \\
\hline
\end{tabular}


arterial en otros sitios vasculares, ya excluidas otras patologías vasculares conocidas). Realiza estudios para valorar trombofilia. El diagnóstico etiológico es discutido por un equipo de especialistas 5 .

- Bolderman et al. Realiza ECG, ecocardiograma transtorácico y Holter para valorar causa cardioembólica. Y realiza panel para valorar trombofilia ${ }^{18}$.

- Yoon et al. Realiza ECG, ecocardiograma y realiza TC multicorte para buscar lesiones arteriales y/o infartos en otros territorios arteriales. No aclara cuál es el estudio para valorar trombofilia ${ }^{27}$.

2. Grupo en que se detalla cómo se evalúa causa cardioembólica: Iga et al (ECG, ecocardiograma transtorácico y transesofágico), Rhee et al (ECG y ecocardiograma) y Mesiano et al (ecocardiograma) $)^{17,19,28}$.

3. Grupo en que refiere búsqueda exhaustiva de la causa del infarto renal pero no está especificado: Bourgault et al y Nagasawa et al., ${ }^{3,29}$.

4. Resto de estudios en que se citan causas etiológicas de los infartos renales basados en la historia clínica o sin especificar cómo se obtuvo el diagnóstico.

Se identificaron 718 de 1.582 pacientes con infartos renales de causa cardioaortoembólica
$(45,4 \%), 253(16 \%)$ debidos a injuria arterial renal, $146(9,2 \%)$ debidos a factores protrombóticos, 79 (5\%) se debían a otras causas y $291(18,4 \%)$ se clasificaron como idiopáticos.

Dentro de los de causa cardioaortoembólica, 542 de 718 pacientes tenían fibrilación auricular $(75,5 \%)$.

Dentro del grupo de otras causas, los infartos renales traumáticos fueron 36 de 79 (45,6\%), los infartos renales postoperatorios fueron 13 $(16,5 \%)$, se observó una causa autoinmune en 7 pacientes $(8,9 \%)$ y dentro de la categoría miscelánea se encontraron 14 pacientes $(17,7 \%)$.

Si se sumara, como se ha hecho en algunas revisiones, al grupo de la injuria arterial renal a los pacientes con infarto renal debido a trauma o secundarios a una cirugía o procedimiento diagnóstico, daría un total de 302 pacientes.

Se destaca que de todas las series, solo 5 no tuvieron a la causa cardioaortoembólica como causa más frecuente. Teniendo 2 series a la injuria arterial renal como más frecuente (Faucon et al y Bourgault et al), 2 series al grupo de otras causas como grupo más frecuente (Wong et al, Bertolotto et al) y una serie al grupo idiopático como más frecuente (Karacabey et al) $3,5,20,30,31$.

En la Tabla 2 se resumen los resultados encontrados.

Tabla 2. Principales resultados de series seleccionadas, ordenadas por fecha de publicación

\begin{tabular}{|c|c|c|c|c|c|}
\hline Autor & Título & Año & Revista & $\begin{array}{l}\text { Diseño/ } \\
\text { Metodología }\end{array}$ & Resultados \\
\hline $\begin{array}{l}\text { Lessman R, Johnson S, } \\
\text { Courn J, Kaufman J }\end{array}$ & $\begin{array}{l}\text { Renal Artery Embolism. } \\
\text { Clinical Features and } \\
\text { Long-Term Follow-up of } \\
17 \text { Cases }\end{array}$ & 1978 & $\begin{array}{l}\text { Annals of } \\
\text { Internal } \\
\text { Medicine }\end{array}$ & Serie de casos & $\begin{array}{l}17 \text { casos incluidos, } 14 \text { con } \\
\text { diagnóstico antemortem } \\
13 \text { de causa cardio- } \\
\text { aortoembólica, } 10 \text { por } \\
\text { fibrilación auricular }\end{array}$ \\
\hline $\begin{array}{l}\text { Winzelberg GG, Hull JD, } \\
\text { Agar JWM, Rose BD, } \\
\text { Pletka PG }\end{array}$ & $\begin{array}{l}\text { Elevation of Serum } \\
\text { Lactate Dehydrogenase } \\
\text { Levels in Renal Infarction }\end{array}$ & 1979 & JAMA & Serie de casos & $\begin{array}{l}5 \text { casos, } 4 \text { debidas a causa } \\
\text { cardio-aortoembólica por } \\
\text { fibrilación auricular }\end{array}$ \\
\hline $\begin{array}{l}\text { Wong W, Moss A, } \\
\text { Federle M, Cochran S, } \\
\text { London S }\end{array}$ & $\begin{array}{l}\text { Renal infarction: } C T \\
\text { diagnosis and correlation } \\
\text { between } C T \text { findings and } \\
\text { etiologies }\end{array}$ & 1984 & Radiology & Serie de casos & $\begin{array}{l}12 \text { casos incluidos, } \\
3 \text { por causa cardio- } \\
\text { aortoembólica, } 2 \text { por } \\
\text { fibrilación auricular }\end{array}$ \\
\hline $\begin{array}{l}\text { Hilton S, Bosniak MA, } \\
\text { Raghavendra BN, } \\
\text { Subramanyam BR, } \\
\text { Rothberg M, Megibow AJ }\end{array}$ & $\begin{array}{l}\mathrm{CT} \text { findings in acute renal } \\
\text { infarction }\end{array}$ & 1984 & $\begin{array}{l}\text { Urologic } \\
\text { Radiology }\end{array}$ & Serie de casos & $\begin{array}{l}12 \text { casos incluidos, } \\
5 \text { por causa cardio- } \\
\text { aortoembólica, } 4 \text { casos } \\
\text { por fibrilación auricular }\end{array}$ \\
\hline $\begin{array}{l}\text { Domanovits H, Paulis } \\
\text { M, Nikfardjam M, } \\
\text { Meron G, Kurkciyan I, } \\
\text { Bankier A, et al }\end{array}$ & $\begin{array}{l}\text { Acute renal infarction. } \\
\text { Clinical characteristics of } \\
17 \text { patients. }\end{array}$ & 1999 & $\begin{array}{l}\text { Medicine } \\
\text { (Baltimore) }\end{array}$ & Serie de casos & $\begin{array}{l}17 \text { casos incluidos, } 14 \text { por } \\
\text { causa cardioaortoembóli- } \\
\text { ca, } 11 \text { debidos a fibrila- } \\
\text { ción auricular }\end{array}$ \\
\hline
\end{tabular}




\begin{tabular}{|c|c|c|c|c|c|}
\hline Autor & Título & Año & Revista & $\begin{array}{l}\text { Diseño/ } \\
\text { Metodología }\end{array}$ & Resultados \\
\hline $\begin{array}{l}\text { Lumerman JH, Hom D, } \\
\text { Eiley D, Smith AD }\end{array}$ & $\begin{array}{l}\text { Heightened Suspicion and } \\
\text { Rapid Evaluation with } \\
\text { CT for Early Diagnosis of } \\
\text { Partial Renal Infarction }\end{array}$ & 1999 & $\begin{array}{l}\text { Journal of } \\
\text { Endourology }\end{array}$ & Serie de casos & $\begin{array}{l}7 \text { casos incluidos, } 7 \\
\text { por causa cardio- } \\
\text { aortoembólica, } 4 \text { debidas } \\
\text { a fibrilación auricular }\end{array}$ \\
\hline $\begin{array}{l}\text { Korzets Z, Plotkin E, } \\
\text { Bernheim J, Zissin R }\end{array}$ & $\begin{array}{l}\text { The clinical spectrum of } \\
\text { acute renal infarction }\end{array}$ & 2002 & $\begin{array}{l}\text { The Israel } \\
\text { Medical } \\
\text { Association } \\
\text { Journal }\end{array}$ & Serie de casos & $\begin{array}{l}11 \text { casos incluidos, } \\
8 \text { por causa cardio- } \\
\text { aortoembólica, } 7 \text { debidos } \\
\text { a fibrilación auricular }\end{array}$ \\
\hline $\begin{array}{l}\text { Bolderman R, Oyen R, } \\
\text { Verrijcken A, Knockaert } \\
\text { D, Vanderschueren S }\end{array}$ & Idiopathic renal infarction & 2006 & $\begin{array}{l}\text { American } \\
\text { Journal of } \\
\text { Medicine }\end{array}$ & Serie de casos & $\begin{array}{l}27 \text { casos incluidos, } \\
11 \text { por causa cardio- } \\
\text { aortoembólica, } 10 \text { debidas } \\
\text { a fibrilación auricular }\end{array}$ \\
\hline $\begin{array}{l}\text { Chu PL, Wei YF, Huang } \\
\text { JW, Chen SI, Chu TS, } \\
\text { Wu KD }\end{array}$ & $\begin{array}{l}\text { Clinical characteristics of } \\
\text { patients with segmental } \\
\text { renal infarction }\end{array}$ & 2006 & Nephrology & Serie de casos & $\begin{array}{l}22 \text { casos incluidos, } \\
14 \text { por causa cardio- } \\
\text { aortoembólica, } 11 \\
\text { debidos a fibrilación } \\
\text { auricular }\end{array}$ \\
\hline $\begin{array}{l}\text { Tsai SH, Chu SJ, Chen } \\
\text { SJ, Fan YM, Chang WC, } \\
\text { Wu CP et al }\end{array}$ & $\begin{array}{l}\text { Acute renal infarction: a } \\
\text { 10-year experience }\end{array}$ & 2007 & $\begin{array}{l}\text { International } \\
\text { journal } \\
\text { of clinical } \\
\text { practice }\end{array}$ & Serie de casos & $\begin{array}{l}18 \text { casos incluidos, } \\
9 \text { por causa cardio- } \\
\text { aortoembólica, } 5 \text { debidos } \\
\text { a fibrilación auricular }\end{array}$ \\
\hline $\begin{array}{l}\text { Bertolotto M, } \\
\text { Martegani A, Aiani L, } \\
\text { Zappetti R, Cernic S, } \\
\text { Cova MA }\end{array}$ & $\begin{array}{l}\text { Value of contrast-en- } \\
\text { hanced ultrasonography } \\
\text { for detecting renal in- } \\
\text { farcts proven by contrast } \\
\text { enhanced CT. A feasibility } \\
\text { study }\end{array}$ & 2007 & $\begin{array}{l}\text { European } \\
\text { Radiology }\end{array}$ & Serie de casos & $\begin{array}{l}27 \text { casos incluidos, } \\
11 \text { por causa cardio- } \\
\text { aortoembólica, } 9 \text { debidos } \\
\text { a fibrilación auricular }\end{array}$ \\
\hline $\begin{array}{l}\text { Huang CC, Chen WL, } \\
\text { Chen JH, Wu YL, Shiao } \\
\text { CJ }\end{array}$ & $\begin{array}{l}\text { Clinical Characteristics } \\
\text { of Renal Infarction in an } \\
\text { Asian Population Chien- } \\
\text { Cheng }\end{array}$ & 2008 & $\begin{array}{l}\text { Annals of the } \\
\text { Academy of } \\
\text { Medicine, } \\
\text { Singapore }\end{array}$ & Serie de casos & $\begin{array}{l}38 \text { casos incluidos, } \\
25 \text { por causa cardio- } \\
\text { aortoembólica, } 17 \\
\text { debidos a fibrilación } \\
\text { auricular }\end{array}$ \\
\hline $\begin{array}{l}\text { Antopolsky M, } \\
\text { Simanovsky N, } \\
\text { Stalnikowicz R, Salameh } \\
\text { S, Hiller N }\end{array}$ & $\begin{array}{l}\text { Renal infarction in the } \\
\text { ED: } 10 \text {-year experience } \\
\text { and review of the } \\
\text { literature }\end{array}$ & 2011 & $\begin{array}{l}\text { American } \\
\text { Journal of } \\
\text { Emergency } \\
\text { Medicine }\end{array}$ & Serie de casos & $\begin{array}{l}38 \text { casos incluidos, } \\
17 \text { por causa cardio- } \\
\text { aortoembólica, } 14 \\
\text { debidos a fibrilación } \\
\text { auricular }\end{array}$ \\
\hline $\begin{array}{l}\text { Rhee H, Song SH, Won } \\
\text { Lee D, Lee SB, Kwak IS, } \\
\text { Seong EY }\end{array}$ & $\begin{array}{l}\text { The significance of } \\
\text { clinical features in the } \\
\text { prognosis of acute renal } \\
\text { infarction: Single center } \\
\text { experience }\end{array}$ & 2012 & $\begin{array}{l}\text { Clinical and } \\
\text { Experimental } \\
\text { Nephrology }\end{array}$ & Serie de casos & $\begin{array}{l}67 \text { casos incluidos, } \\
20 \text { por causa cardio- } \\
\text { aortoembólica, } 17 \\
\text { debidos a fibrilación } \\
\text { auricular }\end{array}$ \\
\hline $\begin{array}{l}\text { Bourgault M, Grimbert } \\
\text { P, Verret C, Pourrat J, } \\
\text { Herody M, Halimi JM, } \\
\text { et al. }\end{array}$ & $\begin{array}{l}\text { Acute renal infarction: A } \\
\text { case series }\end{array}$ & 2013 & $\begin{array}{l}\text { Clinical } \\
\text { Journal of } \\
\text { the American } \\
\text { Society of } \\
\text { Nephrology }\end{array}$ & Serie de casos & $\begin{array}{l}94 \text { casos incluidos, } \\
23 \text { por causa cardio- } \\
\text { aortoembólica, } 17 \\
\text { debidos a fibrilación }\end{array}$ \\
\hline $\begin{array}{l}\text { Bae EJ, Hwang K, Jang } \\
\text { HN, Kim MJ, Jeon DH, } \\
\text { Kim HJ, et al. }\end{array}$ & $\begin{array}{l}\text { A retrospective study } \\
\text { of short- and long- } \\
\text { term effects on renal } \\
\text { function after acute renal } \\
\text { infarction }\end{array}$ & 2014 & Renal Failure & Serie de casos & $\begin{array}{l}100 \text { casos incluidos, } \\
56 \text { por causa cardio- } \\
\text { aortoembólica, } 40 \\
\text { debidos a fibrilación } \\
\text { auricular }\end{array}$ \\
\hline $\begin{array}{l}\text { Karacabey S, Hocagil } \\
\text { H, Sanri E, Hocagil A, } \\
\text { Cuneyt Ardic S, Suman } \\
\text { E, et al. }\end{array}$ & $\begin{array}{l}\text { No suspicion, no disease! } \\
\text { renal infarction: Case } \\
\text { series }\end{array}$ & 2014 & $\begin{array}{l}\text { Urology } \\
\text { Journal }\end{array}$ & Serie de casos & $\begin{array}{l}5 \text { casos incluidos, } \\
2 \text { por causa cardio- } \\
\text { aortoembólica, } 1 \text { debido a } \\
\text { fibrilación auricular }\end{array}$ \\
\hline
\end{tabular}




\begin{tabular}{|c|c|c|c|c|c|}
\hline Autor & Título & Año & Revista & $\begin{array}{c}\text { Diseño/ } \\
\text { Metodología }\end{array}$ & Resultados \\
\hline $\begin{array}{l}\text { Lin WL, Seak CJ, Wu JY, } \\
\text { Weng YM, Chen HC }\end{array}$ & $\begin{array}{l}\text { Risk Factors for Develop- } \\
\text { ment of Chronic Kidney } \\
\text { Disease following Renal } \\
\text { Infarction: Retrospective } \\
\text { Evaluation of Emergency } \\
\text { Room Patients from a } \\
\text { Single Center }\end{array}$ & 2014 & PLOS ONE & Serie de casos & $\begin{array}{l}40 \text { casos incluidos, } \\
18 \text { por causa cardio- } \\
\text { aortoembólica }\end{array}$ \\
\hline $\begin{array}{l}\text { Nagasawa T, Matsuda } \\
\text { K, Takeuchi Y, Fukami H }\end{array}$ & $\begin{array}{l}\text { A case series of acute } \\
\text { renal infarction at a } \\
\text { single center in Japan }\end{array}$ & 2015 & $\begin{array}{l}\text { Clinical and } \\
\text { Experimental } \\
\text { Nephrology }\end{array}$ & Serie de casos & $\begin{array}{l}33 \text { casos incluidos, } \\
24 \text { por causa cardio- } \\
\text { aortoembólica, } 23 \\
\text { debidos a fibrilación } \\
\text { auricular }\end{array}$ \\
\hline $\begin{array}{l}\text { Ongun S, Bozkurt O, } \\
\text { Demir O, Cimen S, } \\
\text { Aslan G }\end{array}$ & $\begin{array}{l}\text { Midterm renal functions } \\
\text { following acute renal } \\
\text { infarction }\end{array}$ & 2015 & $\begin{array}{l}\text { Kaohsiung } \\
\text { Journal of } \\
\text { Medical } \\
\text { Sciences }\end{array}$ & Serie de casos & $\begin{array}{l}23 \text { casos incluidos, } 14 \\
\text { debidos a fibrilación } \\
\text { auricular }\end{array}$ \\
\hline $\begin{array}{l}\text { Oh YK, Yang CW, Kim } \\
\text { Y, Kang S, Park CW, } \\
\text { Kim YS, et al. }\end{array}$ & $\begin{array}{l}\text { Clinical Characteristics } \\
\text { and Outcomes of Renal } \\
\text { Infarction }\end{array}$ & 2016 & $\begin{array}{l}\text { American } \\
\text { Journal } \\
\text { of Kidney } \\
\text { Diseases }\end{array}$ & Serie de casos & $\begin{array}{l}438 \text { casos incluidos, } \\
244 \text { por causa cardio- } \\
\text { aortoembólica, } 211 \\
\text { debidos a fibrilación } \\
\text { auricular }\end{array}$ \\
\hline $\begin{array}{l}\text { Yang J, Lee JY, Na YJ, } \\
\text { Lim SY, Kim MG, Jo SK, } \\
\text { et al. }\end{array}$ & $\begin{array}{l}\text { Risk factors and } \\
\text { outcomes of acute renal } \\
\text { infarction }\end{array}$ & 2016 & $\begin{array}{l}\text { Kidney } \\
\text { Research } \\
\text { and Clinical } \\
\text { Practice }\end{array}$ & Serie de casos & $\begin{array}{l}89 \text { casos incluidos, } 22 \\
\text { debidos a fibrilación } \\
\text { auricular }\end{array}$ \\
\hline $\begin{array}{l}\text { Mesiano P, Rollino } \\
\text { C, Beltrame G, Ferro } \\
\text { M, Quattrocchio G, } \\
\text { Fenoglio R. }\end{array}$ & $\begin{array}{l}\text { Acute renal infarction: a } \\
\text { single center experience }\end{array}$ & 2016 & $\begin{array}{l}\text { Journal of } \\
\text { Nephrology }\end{array}$ & Serie de casos & $\begin{array}{l}18 \text { casos incluidos, } 5 \\
\text { debidos a fibrilación } \\
\text { auricular }\end{array}$ \\
\hline $\begin{array}{l}\text { Kwon JH, Oh BJ, Ha SO, } \\
\text { Kim DY, Do HH. }\end{array}$ & $\begin{array}{l}\text { Renal Complications } \\
\text { in Patients with Renal } \\
\text { Infarction: Prevalence and } \\
\text { Risk Factors }\end{array}$ & 2016 & $\begin{array}{l}\text { Kidney } \\
\text { and Blood } \\
\text { Pressure } \\
\text { Research }\end{array}$ & Serie de casos & $\begin{array}{l}105 \text { casos incluidos, } \\
58 \text { por causa cardio- } \\
\text { aortoembólica, } 50 \\
\text { debidos a fibrilación } \\
\text { auricular }\end{array}$ \\
\hline $\begin{array}{l}\text { Caravaca-Fontán F, } \\
\text { Pampa Saico S, Elías } \\
\text { Triviño S, Galeano } \\
\text { Álvarez C, Gomis Couto } \\
\text { A, Pecharromán de las } \\
\text { Heras I, et al. }\end{array}$ & $\begin{array}{l}\text { Infarto renal agudo: } \\
\text { características clínicas y } \\
\text { factores pronósticos }\end{array}$ & 2016 & Nefrología & Serie de casos & $\begin{array}{l}62 \text { casos incluidos, } \\
30 \text { por causa cardio- } \\
\text { aortoembólica, } 21 \\
\text { debidos a fibrilación } \\
\text { auricular }\end{array}$ \\
\hline $\begin{array}{l}\text { Faucon AL, Bobrie G, } \\
\text { Jannot AS, Azarine A, } \\
\text { Plouin PF, Azizi M. }\end{array}$ & $\begin{array}{l}\text { Cause of renal infarction: } \\
\text { a retrospective analysis of } \\
186 \text { consecutive cases. }\end{array}$ & 2017 & $\begin{array}{c}\text { Journal of } \\
\text { hypertension }\end{array}$ & Serie de casos & $\begin{array}{l}186 \text { casos incluidos, } \\
17 \text { por causa cardio- } \\
\text { aortoembólica, } 8 \text { debidos } \\
\text { a fibrilación auricular }\end{array}$ \\
\hline $\begin{array}{l}\text { Kagaya S, Yoshie O, } \\
\text { Fukami H, Sato H, Saito } \\
\text { A, Takeuchi Y, et al. }\end{array}$ & $\begin{array}{l}\text { Renal infarct volume and } \\
\text { renal function decline in } \\
\text { acute and chronic phases }\end{array}$ & 2017 & $\begin{array}{l}\text { Clinical and } \\
\text { Experimental } \\
\text { Nephrology }\end{array}$ & Serie de casos & $\begin{array}{l}39 \text { casos incluidos, } \\
30 \text { por causa cardio- } \\
\text { aortoembólica }\end{array}$ \\
\hline $\begin{array}{l}\text { Yoon K, Song SY, Lee } \\
\mathrm{CH}, \mathrm{Ko} \mathrm{BH} \text {, Lee S, Kang } \\
\text { BK, et al. }\end{array}$ & $\begin{array}{l}\text { Spontaneous Renal Artery } \\
\text { Dissection as a Cause of } \\
\text { Acute Renal Infarction: } \\
\text { Clinical and MDCT } \\
\text { Findings }\end{array}$ & 2017 & $\begin{array}{l}\text { Journal } \\
\text { of Korean } \\
\text { Medical } \\
\text { Science }\end{array}$ & & $\begin{array}{l}35 \text { casos incluidos, } \\
14 \text { por causa cardio- } \\
\text { aortoembólica, } 5 \text { debidos } \\
\text { a fibrilación auricular }\end{array}$ \\
\hline
\end{tabular}




\section{Discusión}

Esta revisión es la más grande sobre el tema hasta la fecha y se centra en la etiología del infarto renal. La revisión evidencia que la causa principal de infarto renal es la cardioaortoembólica $(45,4 \%)$, y dentro de este grupo la fibrilación auricular abarca el 75,5\%.

Se han reportado diferentes opciones terapéuticas como: anticoagulación, terapia endovascular (trombólisis local, trombectomía o colocación de stent) y cirugía (su rol se limita a infarto renal de causa traumática). No se ha podido establecer el tratamiento óptimo para el infarto renal y dado que entidades subyacentes tienen clara indicación de anticoagulación para evitar nuevas embolias, esta es la razón por la que se debe realizar el máximo esfuerzo para determinar la etiología. La anticoagulación (cuyo objetivo principal es prevenir eventos futuros) está claramente indicada en los casos de fibrilación auricular, trombo del ventrículo izquierdo o una trombofilia. Sin embargo, en otros entornos como la disección aórtica o la endocarditis no está indicada.

Como se ha resaltado en la sección resultados, la causa principal de infarto renal es la cardioaortoembólica y dentro de este grupo predomina la fibrilación auricular. Pero como también se destacó en esa sección hay 5 series en la que la causa cardioaortoembólica no es la más frecuente. De estas, se quiere destacar a las series de Faucon y Bourgault porque tienen un número mayor de pacientes reclutados 186 y 94 respectivamente y en el caso de la serie de Faucon por la baja frecuencia de causa cardioaortoembólica (17 pacientes, 9,1\%) y fibrilación auricular reportadas (8 pacientes, $4,3 \%)$.

\section{¿Cuáles son los motivos de esta discrepancia?}

El primer punto a tomar en cuenta sería la media/mediana de edad de ambas series (Bourgault $52,9 \pm 16,6$ a yaucon 53,3 rango intercuartílico de 42,5-64,5) versus la media de edad de las 15 series que aportan este dato es de 60,33 a, ya que la fibrilación auricular aumenta su prevalencia al aumentar la edad. Y por otra parte, en el trabajo de Bourgault ${ }^{30}$ se observó que el grupo de infarto renal de causa cardioaortoembólica tenía una mayor edad que el de injuria arterial renal.

La segunda causa que podría explicar esta diferencia, sería el sesgo de los pacientes incluidos en el trabajo de Faucon (Francia con $n=186$ ) ya que este abarca pacientes asistidos en una Unidad de Hipertensión especializada en el manejo de lesiones arteriales renales. Sin embargo, el trabajo de Bourgault (Francia con $n=94$ ) incluye pacientes asistidos por los departamentos de nefrología y medicina interna en 14 hospitales no necesariamente especializados en este tipo de lesiones.

Recordando que la mayoría de los pacientes (1.024 de 1.582) provienen de extremo oriente (Corea, Japón y Taiwán), creemos que la diferencia en el porcentaje de casos de fibrilación auricular observados en ambas series francesas no se explicaría por una menor prevalencia de la misma en las distintas regiones (poblaciones caucásicas versus asiáticas). De hecho la fibrilación auricular es más prevalente en los caucásicos que en los afrodescendientes y asiáticos ${ }^{32}$. En una revisión sistemática acerca de la epidemiologia mundial de la fibrilación auricular, la región que incluye a Japón y Corea del Sur fue a nivel mundial la que tuvo menor prevalencia ${ }^{33}$.

Por último, la forma de estudiar a los pacientes podría generar un sesgo. La búsqueda no exhaustiva de la fibrilación auricular podría disminuir su porcentaje. El trabajo de Bourgault refiere que se realizó una búsqueda exhaustiva pero no se detalla cómo. Mientras que en el trabajo de Faucon se detalla de forma clara la búsqueda de ciertas patologías que pueden ser enmarcadas en el grupo de injuria arterial renal y es también una de las 2 series (junto con Bolderman, estudio belga con $n=27$ ) que valora de forma más completa el polo cardioaortoembólico incluyendo un estudio Holter. Sin embargo, el porcentaje de causa cardioaortoembólica es muy diferente 9,1\% (17 pacientes) para Faucon y 40,7\% (11 pacientes) para Bolderman.

Sería conveniente dada la relevancia de encontrar fibrilación auricular como causa del infarto renal, el protocolizar el uso de registros más extensos en el tiempo (como registros de eventos) que se han utilizado para otras patologías como el accidente cerebrovascular ${ }^{34-36}$. El estudio ASSERT buscó la presencia de fibrilación auricular subclínica en individuos mayores de 65 años sin antecedente de fibrilación auricular que utilizaban marcapaso o cardiodesfibrilador implantable. En él se observó que de 2.580 pacientes monitorizados por alrededor de 3 meses, 51 tuvieron un accidente cerebrovascular y de estos 26 (51\%) tuvieron re- 
gistro de fibrilación auricular ${ }^{37}$. Aunque registros tan extensos en el tiempo no serían planteables, un registro más prolongado que el Holter podría ser útil en la detección de fibrilación auricular, hecho que tendría implicancias terapéuticas. Se debe recordar que el diagnóstico de fibrilación auricular aumenta el riesgo relativo de tener embolias arteriales periféricas (entre ellas renales) por 4 veces en hombres y por 5,7 veces en mujeres en comparación con la población general ${ }^{38}$.

Estos estudios más exhaustivos buscando la fibrilación auricular, tal vez darían luz acerca del gran porcentaje de casos que se clasifican como idiopáticos.

Basándose en la forma de estudiar la etiología planteada por Faucon y modificando en parte el protocolo que plantea Lengelé ${ }^{39}$, a continuación se describe un protocolo de estudio de la etiología del infarto renal teniendo en cuenta el mecanismo probable según contexto clínico (Figura 2).

\section{Limitaciones}

La primera limitación de nuestro trabajo dada su condición de revisión, es el sesgo de publicación. Y en segundo lugar, la heterogeneidad en la forma de determinar la etiología entre los diferentes estudios podría influir en los resultados de nuestro trabajo.

\section{Conclusiones}

En la revisión se observó que la principal causa del infarto renal es la cardioaortoembólica y dentro de ésta la fibrilación auricular. Pero todavía 1 de 5 casos se rotulan como idiopáticos. Se debe realizar el máximo esfuerzo diagnóstico para arribar a la etiología del infarto renal ya que esta tiene implicancias terapéuticas. Se propone un protocolo para el estudio de la etiología del infarto renal donde se jerarquiza la búsqueda de fibrilación auricular por su frecuencia y por la indicación de anticoagulación.

\section{Referencias}

1. Hoxie J, Coggin C. Renal infarction statistical study of two hundred and five cases and detailed report of an unusual case. Arch Intern Med 1940; 495: 70-6.

2. Paris B, Bobrie G, Rossignol P, Le S, Chedid A. Blood

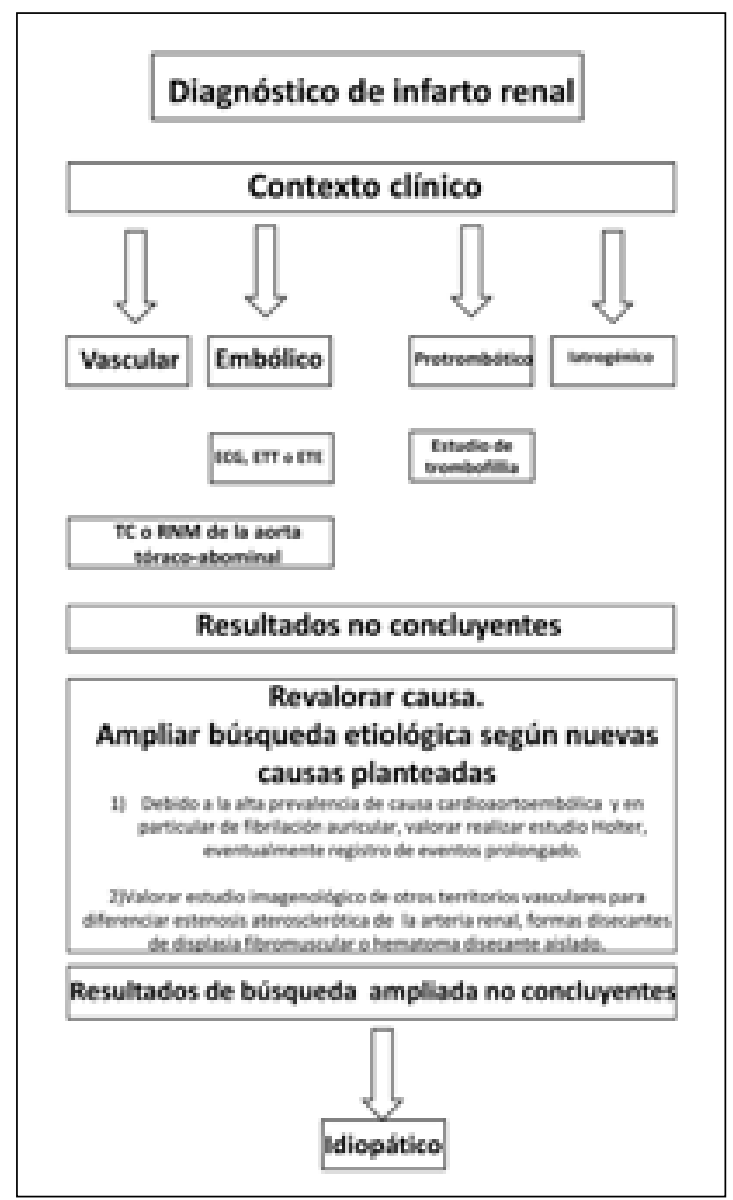

Figura 2. Protocolo de estudio de etiología de infarto renal. Modificado de Lengelé et al. ${ }^{40}$. ECG: electrocardiograma, ETT: ecocardiograma transtorácico, ETE: ecocardiograma transesofágico, TC: tomografía computada, RNM: resonancia nuclear magnética.

pressure and renal outcomes in patients with kidney infarction and hypertension. J Hypertens 2006; 24: 1649-54.

3. Bourgault M, Grimbert P, Verret C, Pourrat J, Herody M, Halimi JM, et al. Acute Renal Infarction: A Case Series.Clin J Am Soc Nephrol 2013; 8 (3): 392-8.

4. Harris D, Jolla L, Hospital T. Tomographic-diagnosis of renal infarction. 1981; XVII (3): 287-9.

5. Faucon A-L, Bobrie G, Jannot A-S, Azarine A, Plouin P-F, Azizi M, et al. Cause of renal infarction: a retrospective analysis of 186 consecutive cases. J Hypertens [Internet]. 2017;1. Available from: http://insights.ovid. com/crossref?an=00004872-900000000-97590\%0Ahttp://www.ncbi.nlm.nih.gov/pubmed/29045340. 
6. Regan FC, Granville Crabtree E. Renal Infarction: A Clinical and Possible Surgical Entity 1. J Urol [Internet]. 1948; 59 (6): 981-1014. Available from: http://linkinghub.elsevier.com/retrieve/pii/S0022534717694758.

7. Heitzman ER, Perchik L. Radiographic features of renal infarction; review of 13 cases. Radiology 1961; 76: 39-46.

8. Hazanov N, Somin M, Attali M, Beilinson N, Thaler M, Mouallem M, et al. Acute renal embolism: Forty-four cases of renal infarction in patients with atrial fibrillation. Medicine (Baltimore) 2004; 83 (5): 292-9.

9. Krämer SC, Seifarth H, Pamler R, Fleiter T, Bühring J, Sunder-Plassmann L, et al. Renal infarction following endovascular aortic aneurysm repair: incidence and clinical consequences. J Endovasc Ther [Internet] 2002; 9 (1): 98-102. Available from: http://www.ncbi.nlm.nih. gov/pubmed/11958332.

10. Böckler D, Krauss M, Mannsmann U, Halawa M, Lange $\mathrm{R}$, Probst T, et al. Incidence of Renal Infarctions after Endovascular AAA Repair: Relationship to Infrarenal versus Suprarenal Fixation. J Endovasc Ther [Internet] 2003; 10 (6): 1054-60. Available from: http://journals. sagepub.com/doi/10.1177/152660280301000605.

11. Janower M, Weber A. Radiologic evaluation of acute renal infarction. Am J Roentgenol Radium Ther Nucl Med 1965; 95 (2): 309-17.

12. Lang EK. Arteriographic diagnosis of renal infarcts. Radiology 1967; 88 (6): 1110-6.

13. Lang EK, Mertz JHO, Nourse M. Renal Arteriography in the Assessment of Renal Infarction. J Urol [Internet] 1968; 99 (5): 506-12. Available from: http://linkinghub. elsevier.com/retrieve/pii/S002253471762740X.

14. Suzer O, Shirkhoda A, Jafri SZ, Madrazo BL, Bis KG, Mastromatteo JF. CT features of renal infarction. Eur J Radiol 2002; 44 (1): 59-64.

15. Romano S, Scaglione M, Gatta G, Lombardo P, Stavolo C, Romano L, et al. Association of splenic and renal infarctions in acute abdominal emergencies. Eur J Radiol 2004; 50 (1): 48-58.

16. Huang $\mathrm{C}$, Lo $\mathrm{H}$, Huang $\mathrm{H}$. ED presentations of acute renal infarction 2007; 164-9.

17. Iga K, Izumi C, Nakano A, Sakanoue Y, Kitaguchi $\mathrm{S}$, Himura $\mathrm{Y}$, et al. Problems in the initial diagnosis of renal infarction. Intern Med [Internet] 1997; 36 (5): 330-2. Available from: http://www.embase.com/ search/results?subaction=viewrecord $\&$ from $=$ export\&i$\mathrm{d}=\mathrm{L} 127281876$

18. Bolderman R, Oyen R, Verrijcken A, Knockaert D, Vanderschueren S. Idiopathic renal infarction. Am J Med 2006; 119 (4): 9-12.

19. Rhee H, Song SH, Won Lee D, Lee SB, Kwak IS, Seong EY. The significance of clinical features in the prognosis of acute renal infarction: Single center experience. Clin Exp Nephrol 2012; 16 (4): 611-6.

20. Bertolotto M, Martegani A, Aiani L, Zappetti R, Cernic $S$, Cova MA. Value of contrast-enhanced ultrasonography for detecting renal infarcts proven by contrast enhanced CT. A feasibility study. Eur Radiol 2008; 18 (2): 376-83.

21. Antopolsky M, Simanovsky N, Stalnikowicz R, Salameh $S$, Hiller N. Renal infarction in the ED: 10-year experience and review of the literature. Am J Emerg Med [Internet] 2012; 30 (7): 1055-60. Available from: http:// dx.doi.org/10.1016/j.ajem.2011.06.041.

22. Caravaca-Fontán F, Pampa Saico S, Triviño SE, Galeano Álvarez C, Gomis Couto A, Pecharromán de las Heras I, et al. Infarto renal agudo: características clínicas y factores pronósticos. Nefrología [Internet] 2016; 36 (2): 141-8. Available from: http://linkinghub.elsevier.com/ retrieve/pii/S0211699515001885.

23. Huang C-C, Chen W-L, Chen J-H, Wu Y-L, Shiao C-J. Clinical characteristics of renal infarction in an Asian population. Ann Acad Med Singapore 2008; 37 (5): 416-20.

24. Kwon JH, Oh BJ, Ha SO, Kim DY, Do HH. Renal Complications in Patients with Renal Infarction: Prevalence and Risk Factors. Kidney Blood Press Res 2016; 41 (6): 865-72.

25. Lessman R, Johnson S, Courn J, Kaufman J. Renal Artery Embolism. Clinical Features and Long-Term Follow-up of 17 Cases. Ann Intern Med 1978; 89 (4): 477-82.

26. Yang J, Lee JY, Na YJ, Lim SY, Kim MG, Jo SK, et al. Risk factors and outcomes of acute renal infarction. Kidney Res Clin Pract [Internet] 2016; 35 (2): 90-5. Available from: http://dx.doi.org/10.1016/j. krcp.2016.04.001.

27. Yoon K, Song SY, Lee CH, Ko BH, Lee S, Kang BK, et al. Spontaneous Renal Artery Dissection as a Cause of Acute Renal Infarction: Clinical and MDCT Findings. J Korean Med Sci [Internet] 2017; 32: 605-12. Available from: http://ovidsp.ovid.com/ovidweb.cgi?$\mathrm{T}=\mathrm{JS} \& \mathrm{CSC}=\mathrm{Y} \& \mathrm{NEWS}=\mathrm{N} \& \mathrm{PAGE}=$ fulltext $\& \mathrm{D}=$ med $1 \& A N=28244286$.

28. Mesiano P, Rollino C, Beltrame G, Ferro M, Quattrocchio G, Fenoglio R, et al. Acute renal infarction: a single center experience. J Nephrol 2017; 30 (1): 103-7.

29. Nagasawa T, Matsuda K, Takeuchi Y, Fukami H. A case series of acute renal infarction at a single center in Japan. Clin Exp Nephrol 2015.

30. Wong W, Moss A, Federle M, Cochran S, London S. Renal infarction: CT diagnosis and correlation between CT findings and etiologies. Radiology 1984; 150: 201-5. 
31. Karacabey S, Hocagil H, Sanri E, Hocagil AC, Ardic S, Suman E. No suspicion, no disease! renal infarction: Case series. Urol J 2014; 11 (6): 1984-6.

32. Dewland TA, Olgin JE, Vittinghoff E, Marcus GM. Incident atrial fibrillation among Asians, hispanics, blacks, and whites. Circulation 2013; 128 (23): 2470-7.

33. Chugh SS, Havmoeller R, Narayanan K, Singh D, Rienstra M, Benjamin EJ, et al. Worldwide epidemiology of atrial fibrillation: A global burden of disease 2010 study. Circulation 2014; 129 (8): 837-47.

34. Svennberg E, Engdahl J, Al-Khalili F, Friberg L, Frykman $\mathrm{V}$, Rosenqvist M. Mass screening for untreated atrial fibrillation the STROKESTOP study. Circulation 2015; 131 (25): 2176-84.

35. Healey J. Subclinical Atrial Fibrillation in Older Patients. Circulation 2017; 136 (14): 1276.
36. Healey J, Connolly S, Gold M, Israel W, Van Gelder I, Capucci A, et al. Subclinical Atrial Fibrillation and the Risk of Stroke Ne 2013; 366: 120-9.

37. Brambatti M, Connolly SJ, Gold MR, Morillo CA, Capucci A, Muto C, et al. Temporal relationship between subclinical atrial fibrillation and embolic events. Circulation 2014; 129 (21): 2094-9.

38. Frost L, Engholm G, Johnsen S, Møller H, Henneberg E, Husted S. Incident Thromboembolism in the Aorta and the Renal, Mesenteric, Pelvic, and Extremity Arteries After Discharge From the Hospital With a Diagnosis of Atrial Fibrillation. Arch Intern Med 2001; 161: 272-6.

39. Lengelé J-P, Christophe J-L, Persu A. Renal infarction management. J Hypertens [Internet] 2018; 36 (3): 490-2. Available from: http://insights.ovid.com/crossref?an $=00004872-201803000-00007$. 